\title{
New Technique for Measuring and Controlling the Permeability of Polymeric Membranes
}

\author{
M. M. El-Tonsy \\ Physics Department \\ Faculty of Science \\ Mansoura University \\ Mansoura 35516, Egypt
}

\author{
I. M. Fouda \\ Physics Department \\ Faculty of Science \\ Mansoura University \\ Mansoura 35516, Egypt
}

\author{
M. A. Farid \\ Physics Department \\ Faculty of Science \\ Mansoura University \\ Mansoura 35516, Egypt
}

\begin{abstract}
Membranes have wide uses in industry and medicine applications. Polymer membranes are important materials because of their high chemical resistance, but they are of weak mechanical resistance against high pressures. Therefore, it was essential to modify a permeability measuring technique free from high pressure application. The current work represented a modification for the permeability measuring technique of membranes, where ionic salt was added with known concentration to water as common solvent and the electrolyte current was measured behind the membrane. The electrolysis current was correlated to the flow rate of water across a polyvinyl alcohol (PVA) membrane. Some other problems were raised such that polarization on electrodes and changes in electrolyte contents during the long time of the slow process. Pulsed potential on electrodes resolved these problems and other associated problems like rush in current and the double layer capacitance effect. An empirical equation was suggested to evaluate the permeability of polymer membranes by this modified method. Easy and accurate measurement of permeability helped authors to change the permeability of PVA membranes by adding copper nano particles in membrane to reduce its permeability, and adding silicone dioxide micro particles to the PVA membranes to increase its permeability. Authors suggested a mechanism for these permeability changes. Scanning electron microscope images for the filled PVA membranes supported the suggested mechanism.
\end{abstract}




\section{INTRODUCTION}

Membrane operation or membrane processes may be regarded as a unit operation in chemical engineering. They are widely used in industrial applications in different fields like chemicals, food, gas, water and wastewater treatment, pharmaceuticals and more. Membrane systems are based on the use of synthetic membranes that permit the concentration and separation of solutions without thermal damage. Particles are separated on the basis of their molecular size and shape using pressure and specially designed synthetic and semi-permeable membranes [1].

Membrane processes provide efficiency and operational simplicity, high selectivity and permeability for the transport of certain components. It is possible to achieve high compatibility as between different membrane operations as between membrane operations and traditional industrial

applications in integrated systems. In order to analyze their economic impact, it is necessary for membrane processes to be characterized by low operational energy, good stability under operative conditions, environment compatibility, easy scale-up, great flexibility, and good control of effectiveness with the possibility of reaching advanced levels of automation and remote control [1]. The most important property of membranes is their ability to control the rate of permeation of different species [2]. The permeability is a measure of the ability of a porous medium to transmit fluids measured in the units of length square or darcies $\left(1 \mathrm{D}=0.98692 \times 10^{-12} \mathrm{~m}^{2}\right)[3]$.

Two models used to describe the mechanism of permeation. One of them is the solution-diffusion model, in which permeates dissolve in the membrane material and then diffuse through the membrane down a concentration gradient. Permeates are separated because of the differences in the solubility of the materials in the membrane and the differences in the rates at which the materials diffuse through the membrane [2]. If a concentration gradient of permeate molecules is formed in the medium, simple statistics show that a net transport of matter will occur from the high concentration to the low concentration region. For example, when two adjacent volume elements with slightly different permeate concentrations are separated by an interface, then simply because of the difference in the number of molecules in each volume element, more molecules will move from the concentrated side to the less concentrated side of the interface than will move in the other direction. This concept was first recognized by Fick theoretically and experimentally [2]. In
1856, Darcy investigated the flow of water through sand filters for water purification [4].

By empirical observation Darcy noticed that fluid flow was directly proportional to the hydraulic gradient [4], resulting in the following equation

$$
q=K A \frac{h_{2}-h_{1}}{l}
$$

where $q$ represents the volumetric flow rate of water downward through the cylindrical sand pack of cross-sectional area $A$ and height $l, h_{1}$ and $h_{2}$ are the hydraulic head above the standard datum of the water in the manometer located at the input and output ports respectively, and $K$ is a constant of proportionality found to be characteristic of the rock media. Most of the techniques for measuring permeability are based on Darcys equation where pressure $\left(h_{2}-h_{1}\right)$ is essential parameter. Many polymeric membranes are deformed under pressure, and hence their surface areas are changed releasing markedly error. Therefore it is essential to develop the measuring technique in order to measure the coefficient of permeability for thin polymeric membranes with satisfactorily degree of accuracy.

The current work aims to design, setup and use a modified measuring system for permeability of a thin polymer membrane and then use this system as a very helpful technique for measuring the change in permeability of polymeric membranes.

\section{THEORETICAL PRINCIPLE}

To achieve the goals described in the previous section we start by designing the system which we going to use in our study. The main idea is to detect and demonstrate the process of permeability and diffusion through simple electrochemical reaction. To do this we going to design a system consist from two chambers, in the first one of them an electrolyte solution is contained and the second one a free of ion solution is contained. The membrane under investigation is held in between of them. In this situation the system is kept under equilibrium and the static pressure on both sides of the membrane is the same where $H_{1}=$ $H_{2}$. The system is illustrated in figure (1). 


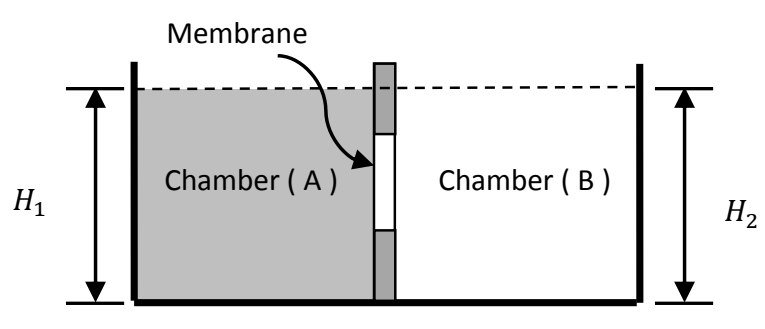

Figure (1): Schematic structure of the main unit

At the beginning of this process there will be no flow expected through the membrane due to the time consumed in the swilling and wicking processes inside the membrane [5]. The time duration consumed in these processes is related to the physical properties of the membrane under investigation. After a while and according to the ficks law of diffusion, the electrolyte starts to flow from chamber (A) which is high in concentration to chamber (B) which have the lower concentration and this will be steady-state flow. As the electrolyte in the chamber (B) starts to reach saturation, the process of diffusion starts to slow down. The expected behavior of the above process is described in figure (2).

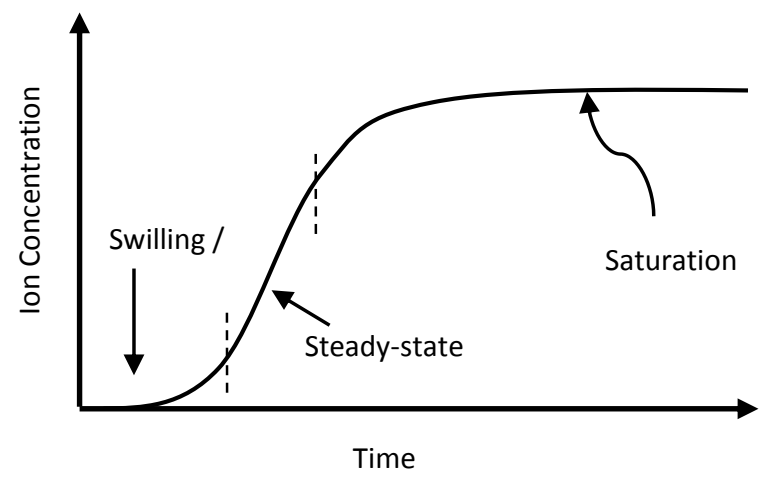

Figure (2): General behavior of the concentration change in chamber B

\section{EXPERIMENTAL}

The main idea on which the suggested technique was designed depends on the electrolysis phenomenon. On this base it was possible to measure both of molar and ionic permeability's of a polymer membrane by any ionic bonds compound.

\section{Setup of the suggested measuring system:}

As shown in figure (3A) the experimental setup is consisting from an external container which will be referred as the electrolyte container and an internal container which will be referred as the distilled water container. The Distilled water container is placed inside the electrolyte container. The circuit board which will be referred as the electrode PCB is attached to the distilled water container and connected to the DAQ device which is connected to the PC.
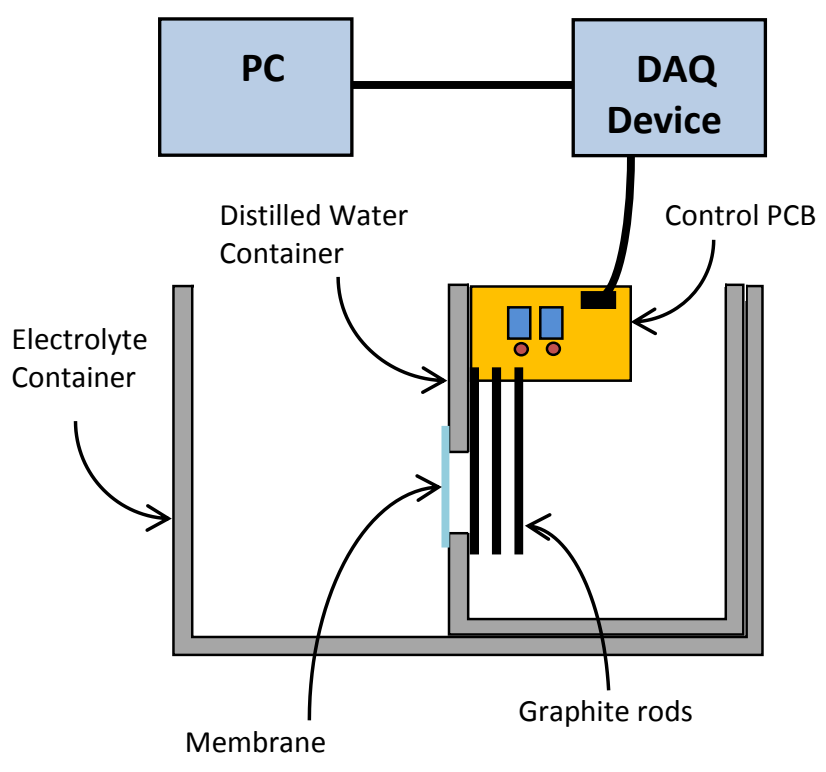

Figure (3A): Schematic diagram for the real used system

The electrolyte container is made of grade A transparent acrylic sheets of $10 \mathrm{~mm}$ thickness and internal dimensions of (25 $\mathrm{cm} \mathrm{W} \times 18 \mathrm{~cm} \mathrm{D} \mathrm{x} 19.5 \mathrm{~cm} \mathrm{H})$ the resistivity of the acrylic is about $10^{15} \mathrm{ohm} / \mathrm{cm}$ for $6 \mathrm{~mm}$ thickness sheets and dielectric strength of $30 \mathrm{Mv} / \mathrm{m}$. Acrylic sheets are resistant to most chemicals, can be cleaned easily and corrosion resistant. So it's very suitable for use in our system. The container is approved to be leak free. The distilled water container is constructed from the same materials used for the electrolyte container with internal dimensions of ( $16 \mathrm{~cm} \mathrm{~W} \mathrm{x} 12.5 \mathrm{~cm} \mathrm{D} \mathrm{x} 20 \mathrm{~cm} \mathrm{H}$ ). The front side of the container is equipped with circular hole of diameter 4.5 $\mathrm{cm}$, a rubber ring is circulating the hole at distance $5 \mathrm{~mm}$ from the hole contour. Four small holes of diameter $4 \mathrm{~mm}$ are drilled around the hole for membrane holder fixation. Four stainless steel screws and bolts are installed and fixed in position using waterproof epoxy to prevent leakage of the electrolyte through 
the screws opening. Figure (3B) shows the membrane fixation mechanism.

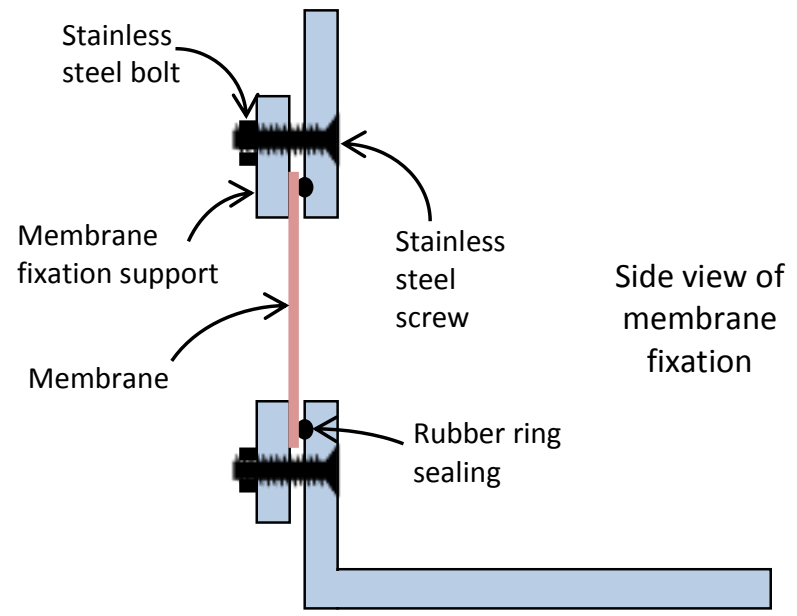

Figure (3B): Fixation of the membrane

The circuit board is assembled on a PCB with an additional depolarization relay. The depolarization relay function is to depolarize the graphite electrodes by applying a charge for certain period of time in the way that the applied polarities on the electrodes are reversed from the applied one during current measurement. Note that this time period must be equal or less than the period used during current measurement. The purpose of adding this relay on the circuit is to minimize the effect of electrodes polarization in the case of long term measurements that extends for several days. Figure (3C) shows the circuit.

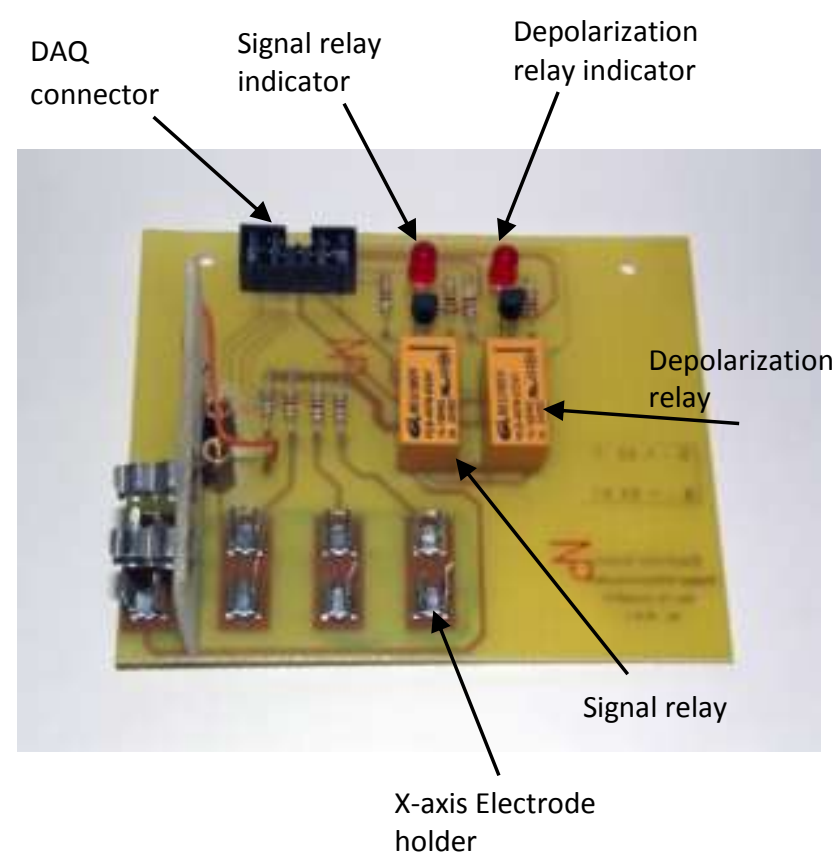

Figure (3C): Electrodes holder and electronic control circuit

\section{System design requirements:}

\section{- Electrode choices:}

Electrode choice is very important decision in system design. The electrodes should satisfy the following specifications:

a) Very low resistance so it can detect very low currents as low as $10 \mu \mathrm{A}$

b) It should be inert electrode which means that the electrode material should not react with any of the reacting species.

c) The electron transfer step is the only reaction step. Which means that other parallel or consecutive steps are absent

According to the above requirements, graphite seems to be the perfect electrode for our mission. Graphite typical resistivity range of $2.5-5 \times 10^{-6} \Omega . \mathrm{m}$ at $20^{\circ} \mathrm{C}$ and conductivity range of $2-3 \times 10^{5} \mathrm{~S} / \mathrm{m}$ at $20^{\circ} \mathrm{C}$ [6]. Graphite is perfect electrical conductor, even with the commercial grades which have higher resistivity and lower conductivity, the above values of resistivity still in the range of $\mu \Omega . m$ which is very accepted in our system and sill verify our design requirements.

\section{- Measuring the current through Voltage divider} resistor:

Current measurements are troublesome, because the current appears within a loop and the loop must be opened to insert the measuring instrument. Moreover, this instrument contains a characteristic resistance or impedance that most often changes the circuit parameters and must be compensated to obtain accurate measurements.

This problem can be eliminated by using voltage divider method. Voltage drops are relatively easy to measure with most instruments because the difference in voltage appears between any two points in a circuit. The circuit does not need to be disturbed or changed [7].

The known reference resistor should have a value that is close to the unknown value. A low current as $1.0 \mu \mathrm{A}$ or less can be measured accurately.

\section{- Electrolyte and solvent choice :}


The first requirement of an electrolyte is that it should give rise to a highly

Conducting solution. Potential electrolytes (organic acids and most

bases) dissociate into ions by ionogenic, or ion-forming, chemical reactions with solvent molecules, in contrast to true electrolytes, which often give rise to ionic solutions by physical interactions between ions present in the ionic crystal and solvent molecules. Sodium chloride which is a true electrolyte dissolves by the solvation of $\mathrm{Na}^{+}$and $\mathrm{Cl}^{-}$ions in the salt crystals.

\section{Observed problems during sysem operation:}

The fact that system uses the hydrodynamic pressure as a working princible results in long time measurement reaching several days. Due to this long time measurement two problemes affecting the accuracy of the recoreded data are observed. The first problem is the polarization of the graphite electrodes and the second problem is the change in the electrolyte content by consuming the ions during the electrolysis. These problem have been resolved by using the pulsed potential technique as will be discussed later on.

\section{Membrane preparation:}

5 gm of PVA (Poly Vinyl Alcohol) from LOBA CHEMIE dissolved in $100 \mathrm{ml}$ of distilled water, the solution placed on magnetic stirrer for 12 hours at $75^{\circ} \mathrm{C}$, the beaker is covered with aluminum foil to minimize the evaporation.

The solution was casted onto glass petri dishes of diameter 70 $\mathrm{mm}$. The casted volume is $10 \mathrm{ml}$ in each petri dish. The solution let to dry for several days at room temperature (about $25^{\circ} \mathrm{C}$ ) until complete dryness. Then the films were peeled off from the dishes. The peeled films are identical in dimensions with thickness of $0.15 \mathrm{~mm}$.

\section{Electrolyte preparation:}

An electrolyte solution of volume 3 liters at the target concentration is prepared by dissolving sodium chloride from BIO BASIC CANADA INC. (MW 58.44, Purity >99.5\%, sulfate $<0.001 \%$ ) into distilled water. The solution is left for $1-2$ hours for complete dissolution.

\section{RESULT AND DISCUSSION}

\section{Electrolysis of Aqueous $\mathrm{NaCl}$ :}

Figure 4 below shows an idealized drawing of a cell in which an aqueous solution of sodium chloride is electrolyzed.

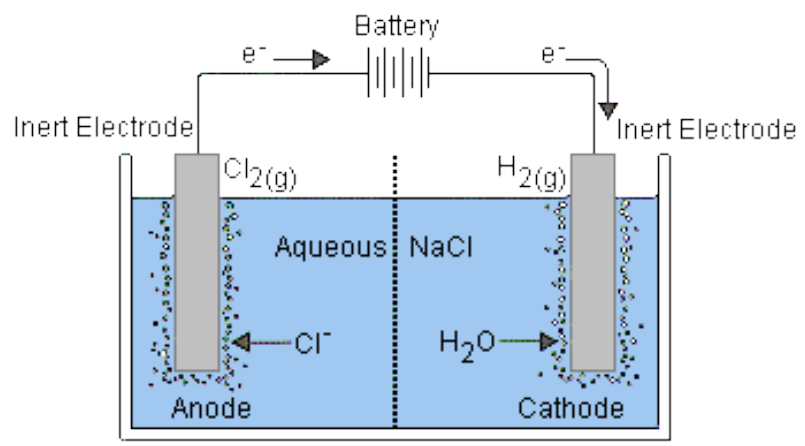

Figure (4): Ideal cell for aqueous $\mathrm{NaCl}$ solution.

The $\mathrm{Na}^{+}$ions migrate toward the negative electrode and the $\mathrm{Cl}^{-}$ ions migrate toward the positive electrode. But, now there are two substances that can be reduced at the cathode: $\mathrm{Na}^{+}$ions and water molecules.

At the Cathode (-):

$$
\begin{aligned}
& \mathrm{Na}^{+}+e^{-} \rightarrow \mathrm{Na} \quad E_{\text {red }} \\
& =-2.71 \mathrm{~V} \\
& 2 \mathrm{H}_{2} \mathrm{O}+2 e^{-} \rightarrow \mathrm{H}_{2}+2 \mathrm{OH}^{-} \quad E_{\text {red }} \\
& =-0.83 \mathrm{~V}
\end{aligned}
$$

Because it is much easier to reduce water than $\mathrm{Na}^{+}$ions, the only product formed at the cathode is hydrogen gas.

The cathode reaction will be:

$$
2 \mathrm{H}_{2} \mathrm{O}+2 e^{-} \rightarrow \mathrm{H}_{2}+2 \mathrm{OH}^{-}(a q)
$$

There are also two substances that can be oxidized at the anode: $\mathrm{Cl}^{-}$ions and water molecules.

\section{At the Anode (+):}

$$
\begin{aligned}
& 2 \mathrm{Cl}^{-} \rightarrow \text { 回 } \mathrm{Cl}_{2}+2 e^{-} \\
& E_{o x}=-1.36 \mathrm{~V} \\
& 2 \mathrm{H}_{2} \mathrm{O} \rightarrow \mathrm{O}_{2}+4 \mathrm{H}^{+}+4 e^{-} \\
& E_{\text {ox }}=-1.23 \mathrm{~V}
\end{aligned}
$$

The standard-state potentials for these half-reactions are so close to each other that we might expect to see a mixture of $\mathrm{Cl}_{2}$ and $\mathrm{O}_{2}$ 
gas collect at the anode. In practice, the only product of this reaction is $\mathrm{Cl}_{2}$.

The anode reaction will be:

$$
2 \mathrm{Cl}^{-} \rightarrow \mathrm{Cl}_{2}+2 e^{-} \quad E_{\text {ox }}=-1.36 \mathrm{~V}
$$

At first glance, it would seem easier to oxidize water $\left(E_{o x}=-1.23\right.$ volts) than $\mathrm{Cl}^{-}$ions ( $E_{o x}=-1.36$ volts). The solution concentration of $\mathrm{NaCl}$ controls the potential required to oxidize the $\mathrm{Cl}^{-}$ion. The deciding factors are:

1- A phenomenon which is known as overvoltage: it is the extra voltage that must be applied to a reaction to aid it to occur at the rate, at which it would occur in an ideal system. The measured overpotential required for oxygen oxidation on the graphite electrodes is $0.95 \mathrm{~V}$ (table 1). Hence chlorine gas is formed over hydrogen gas.

2- The $\mathrm{NaCl}$ concentration: At the cathode the same reduction reaction occurs in both dilute and concentrated solutions.

For the concentrated electrolyte chlorine gas and sodium hydroxide are formed at the anode.

Table (1): Over-potential for different electrodes

\begin{tabular}{|l|l|l|l|}
\hline $\begin{array}{l}\text { Material of } \\
\text { the } \\
\text { electrode }\end{array}$ & Hydrogen & oxygen & Chlorine \\
\hline $\begin{array}{l}\text { Platinum } \\
\text { (platinized) }\end{array}$ & $-0.07 \mathrm{~V}$ & $+0.77 \mathrm{~V}$ & $+0.08 \mathrm{~V}$ \\
\hline Palladium & $-0.07 \mathrm{~V}$ & $+0.93 \mathrm{~V}$ & \\
\hline Gold & $-0.09 \mathrm{~V}$ & $+1.02 \mathrm{~V}$ & \\
\hline Iron & $-0.15 \mathrm{~V}$ & $+0.75 \mathrm{~V}$ & \\
\hline Platinum (shiny) & $-0.16 \mathrm{~V}$ & $+0.95 \mathrm{~V}$ & $+0.10 \mathrm{~V}$ \\
\hline Silver & $-0.22 \mathrm{~V}$ & $+0.91 \mathrm{~V}$ & \\
\hline Nickel & $-0.28 \mathrm{~V}$ & $+0.56 \mathrm{~V}$ & \\
\hline Graphite & $-0.62 \mathrm{~V}$ & $+0.95 \mathrm{~V}$ & $+0.12 \mathrm{~V}$ \\
\hline Lead & $-0.71 \mathrm{~V}$ & $+0.81 \mathrm{~V}$ & \\
\hline Zinc & $-0.77 \mathrm{~V}$ & & \\
\hline Mercury & $-0.85 \mathrm{~V}$ & & \\
\hline
\end{tabular}

External resistor value and electrode potential dependence:

The electrolyte resistance can be considered as a fixed value ohmic resistor for fixed value of electrolyte concentration [8].

The main power supply voltage will be shared between the electrodes and the voltage divider resistor as indicated by the following diagram (Figure 5).

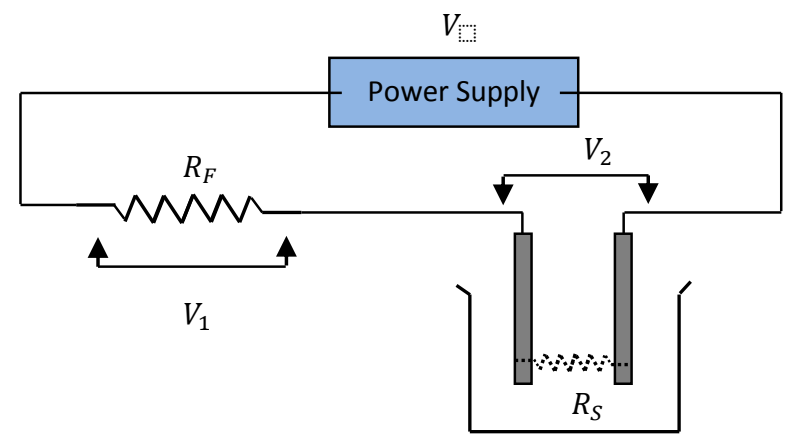

Figure (5): The position of the external resistor in the circuit

From the above circuit we can see that

$$
V=V_{1}+V_{2}
$$

Where;

$V \quad$ is the power supply applied voltage

$V_{1} \quad$ is the voltage across the fixed resistor $R_{F}$

$V_{2}$ is the voltage across the two electrodes immersed in the electrolyte.

The selected maximum value for the power supply is $+5 \mathrm{~V}$.

A setup to simulate the process has been installed using a beaker filled with electrolyte solution of $\mathrm{NaCl}$ of concentration $2 \mathrm{wt} \%$. Using two graphite electrodes the current allowed to flow through the electrolyte. Six different values of the fixed voltage divider resistors are used as follow:

$10 \mathrm{~K} \Omega, 4.7 \mathrm{~K} \Omega, 2.6 \mathrm{~K} \Omega, 1 \mathrm{k} \Omega, 510 \Omega, 51 \Omega$

The voltage from the power source is set to increase from zero to $+5 \mathrm{~V}$ in steps of $0.1 \mathrm{~V}$ per minute with the aid of software timed program. The obtained voltage behavior for $10 \mathrm{k}, 1 \mathrm{k}$, and $51 \mathrm{ohm}$ resistors are shown in figures $6,7,8$ respectively while the current behavior for all the resistors is show in figure (9). 


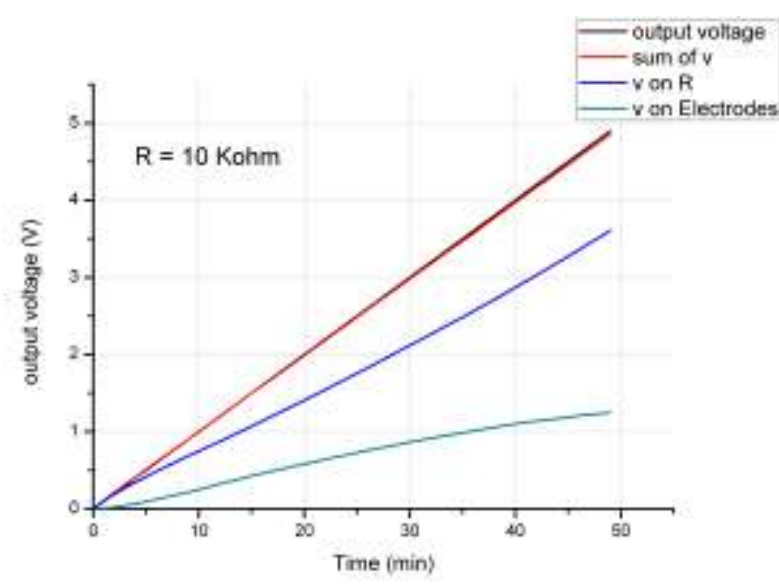

Figure (6): Voltages for external resistance of $10 \mathrm{k} \Omega$

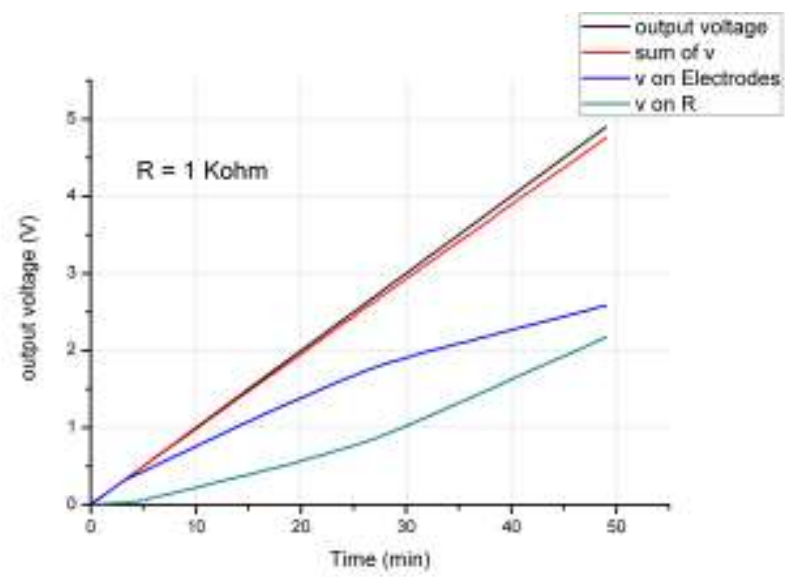

Figure (7): Voltages for external resistance of $1 \mathrm{k} \Omega$

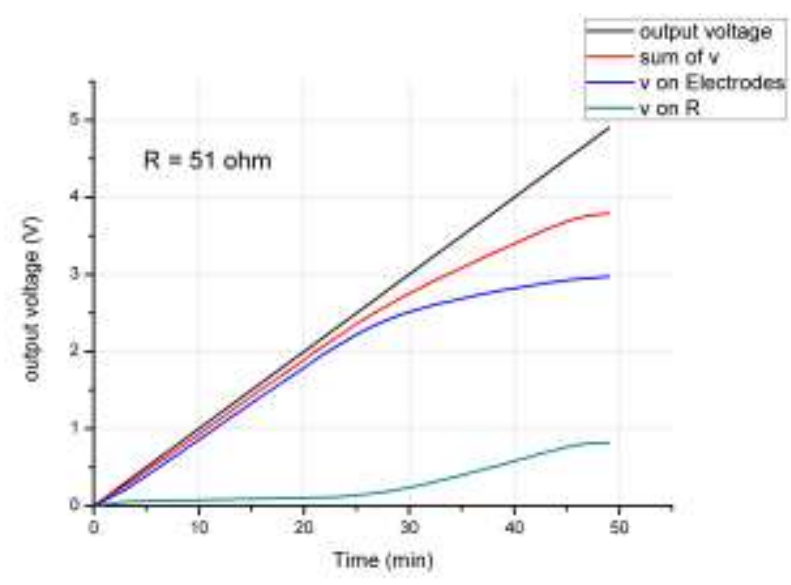

Figure (8): Voltages for external resistance of $51 \Omega$

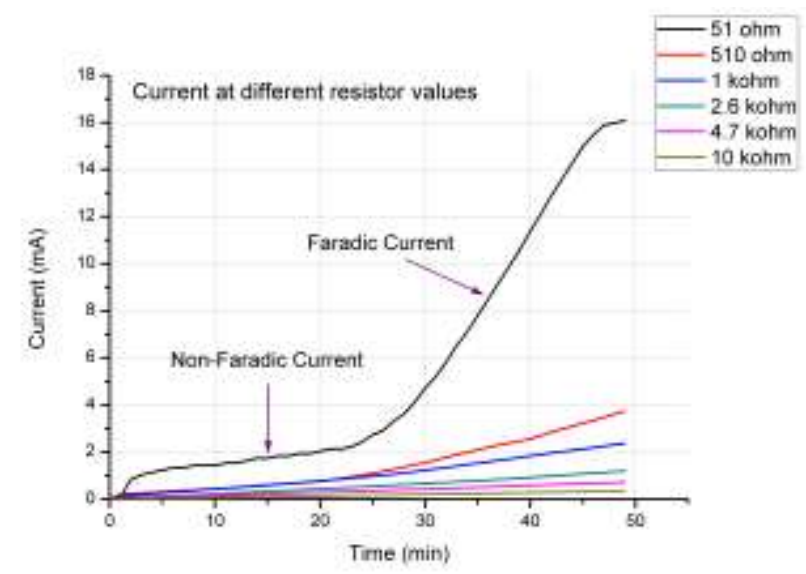

Figure (9): I(t) for different external resistors

The use of power source with value of $+5 \mathrm{~V}$ is appropriate specially when using low voltage divider resistor values. The resistor of value $51 \Omega$ is typical to be used, showing appropriate values of current to be measured and perfect linearity with voltage in the conducting faradic region.

Note that when using a power source of $+5 \mathrm{~V}$ the region corresponding to voltage values from 0 to $2.5 \mathrm{~V}$ (from 0 to 25 minute in figure (9)) will be neglected since minimal number of ions will be enough to produce a faradic current. The nonfaradic current region in figure (9) effect will be minimal since a limited no of ions will transfer the current from non-faradic to the faradic region.

\section{Polarization of Electrodes:}

In figure (8) ( where the greater voltage deviation occurred ) we can relate the value of deviation by the amount of current as seen in figure (9) where the recorded sum values starts to decrease as the current start to increase. Since the fixed resistor can't show such behavior we can assume that the phenomenon is related to the electrodes [9]. The reduced value of voltage on the electrode is caused by voltage drop inside the electrodes causing the measured value to be lower than expected, this phenomena is called "polarization" of electrodes and is varied by: Current value, applied voltage, electrode material and duration of current flow. The magnitude of polarization is more effective by the increasing of any one of the previous four factors. 


\section{The effect of long time current flow:}

In this experiment we going to simulate the best value obtained for power supply voltage which is $5 \mathrm{~V}$ for long period of time.

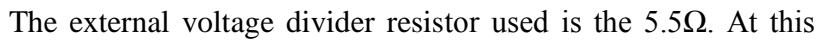
value as seen in figure (10) the power supply voltage is kept at the $+5 \mathrm{v}$ value during the whole 42 hours of the test, and the corresponding current value reaches more than $180 \mathrm{~mA}$. The voltage on the electrodes exceeds $4 \mathrm{~V}$ which is ideal for the electrolysis process. The voltage on the fixed resistor is about $1 \mathrm{~V}$ and the polarization value is minimal.

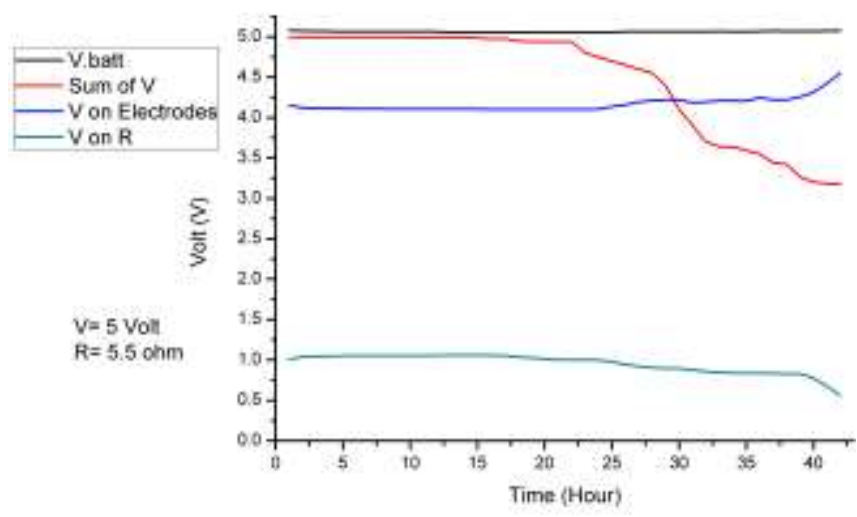

Figure (10): The polarization effect in the electrodes.

As seen in figure (10) in the first 20 hours of the test all the values of power source, polarization, voltage on electrodes and voltage on the fixed resistor are showing linear behavior. But after that time the measured values starts to deviate. While the power supply voltage value is kept stable and fixed, all other values are completely changed. A major voltage drop in the polarization curve is observed.

The current is started to decrease gradually (as seen in figure 11) and this decrease in the current is combined with increase in the solution resistance.

The unexpected deviation from the regular behavior is due to the change in the electrolyte content due to the decrease in the electrolyte ion content and hence increased solution resistance. In conclusion the DC current value recorded in the electrolysis process can be related to the electrolyte concentration only for fast permeation process.

In low permeability membranes and at low concentration the obtained current values will be inaccurate.

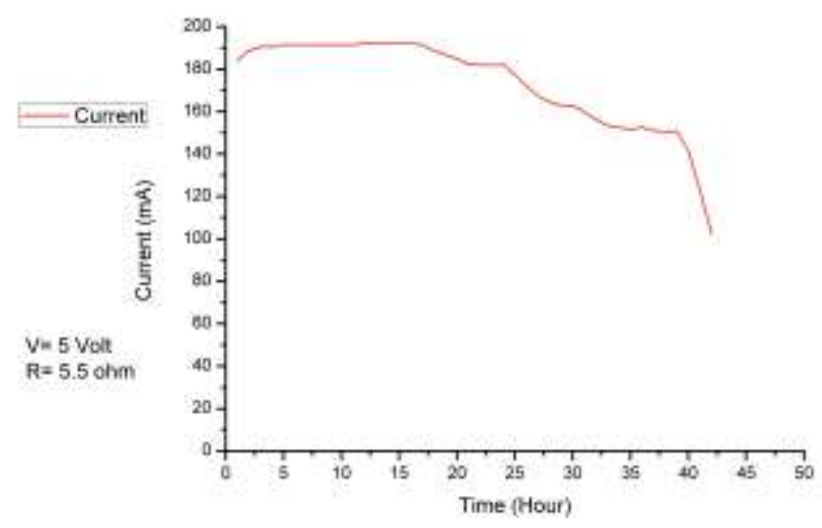

Figure (11): The electrolyte current shows the increase in solution resistance

\section{Pulsed potential technique:}

The suggested modified method is focusing on reducing the time that the current allowed to pass through the electrolyte by substituting the continuous potential application on the electrodes by a pulsed potential for a short period of time, thus reducing the effect of electrode polarization and conserving the electrolyte content (Figure 12).

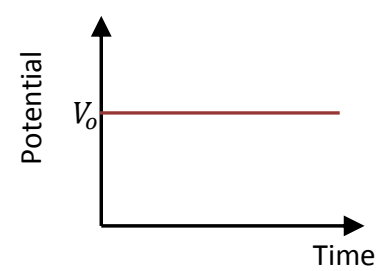

(a)

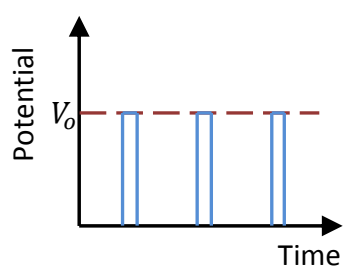

(b)
Figure (12): (a) continuous application of potential $V_{o}$, (b) pulsed potential application with the same $V_{o}$ amplitude.

To achieve our goal the cell design must be modified. The following circuit was used to control the cell potential according to the new enhancements (Figure 13). 


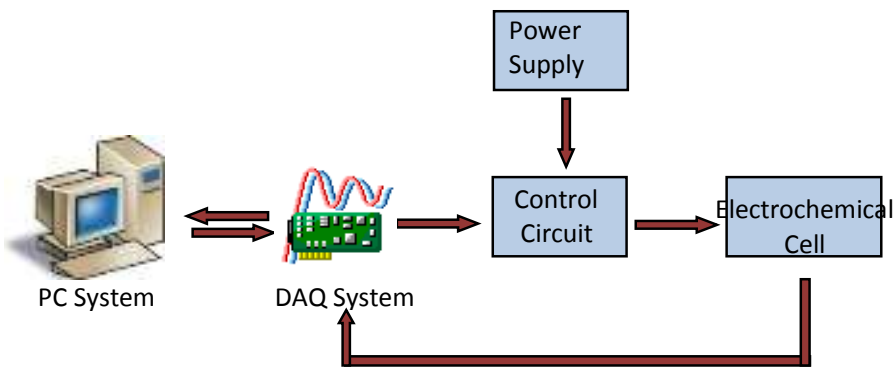

Figure (13): modified measuring system.

The control circuit is demonstrated in figure (14).

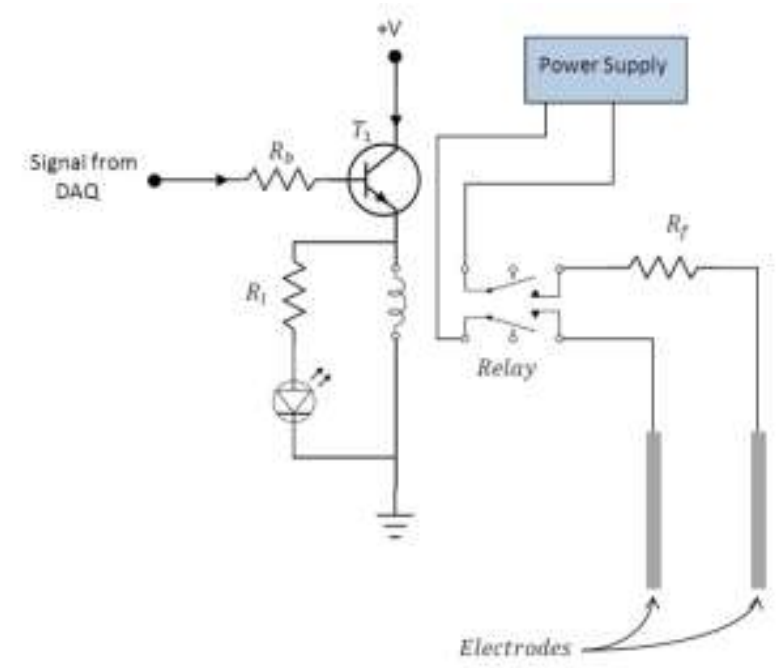

Figure (14): The suggested control circuit.

In the above circuit, the signal coming from the DAQ device is fed to the transistor $T_{1}$ through the biasing resistor $R_{b}$ to trigger the relay.

The DAQ device controls the time periods $T_{o n}$ and $T_{o f f}$ to form the shape of the applied potential pulse.

However this new technique is combined with two new problems associated with the applied pulse technique, which is formation of the rush in current effect and the double layer capacitance effect. Figure (15a) shows the applied pulsed signal, the resultant signal due to the effect of the double layer capacitance is shown in figure (15b). Figure (16) shows the effect of rush in current and the minimum required pulse width.

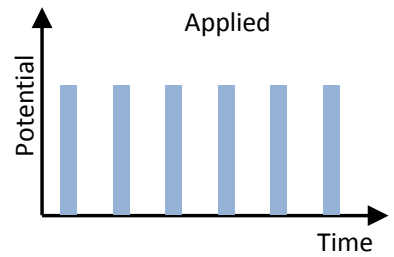

(a)

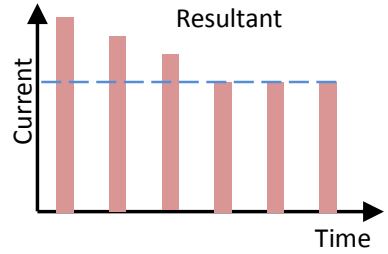

(b)
Figure (15): Applied pulses (a) and output current affected by the double layer capacitance (b)

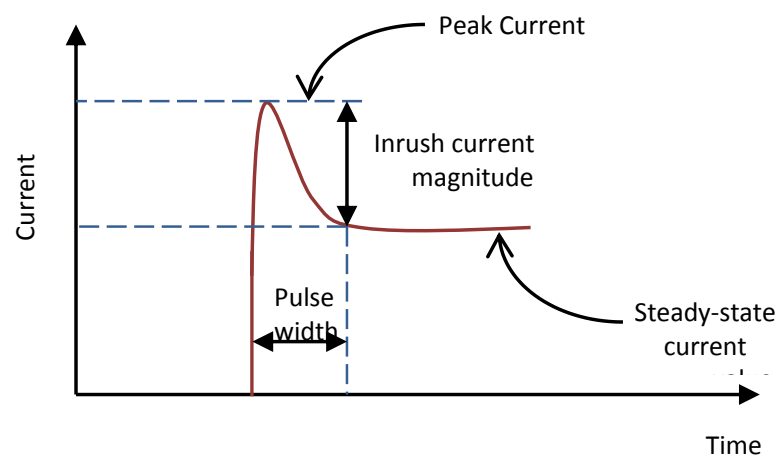

Figure (16): The effect of rush in current on the output current

It's found by experimental trials and measurements that pulse separation above 10 second shows no double layer capacitance effect. Also the pulse width of 3 second was enough to eliminate the effect of the rush in current. Figure (17) show the relation between the applied pulse width and the percentage deviation from the corresponding DC value.

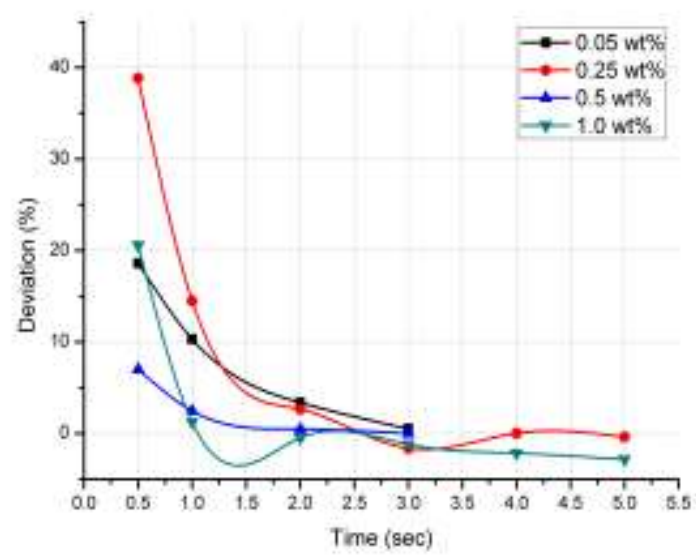

Figure (17): Percentage deviation from DC Value 


\section{Permeability investigation using different concentrations:}

This test was repeated several times using identical PVA films with the same dimensions, the only changed factor is the electrolyte concentration. In each test the flow rate is calculated under the steady state condition of flow. The rate is obtained as increase in current by time and is recorded in $\mathrm{mA} / \mathrm{H}$ (milliampere per hour) values. Table (2) concludes the obtained flow rates for every concentration used.

Table (2): The flow rate at different concentrations

\begin{tabular}{|c|c|}
\hline Concentration (wt \%) & Flow rate (mA/h) \\
\hline $\mathbf{0 . 0 3 3}$ & 0.035 \\
\hline $\mathbf{0 . 0 6 6}$ & 0.054 \\
\hline $\mathbf{0 . 1 3 3}$ & 0.0999 \\
\hline $\mathbf{0 . 2 6 6}$ & 0.229 \\
\hline $\mathbf{0 . 5 3 3}$ & 0.61 \\
\hline $\mathbf{1 . 0 6 6}$ & 1.09 \\
\hline $\mathbf{2 . 1 3 3}$ & 2.013 \\
\hline $\mathbf{3 . 2}$ & 2.49 \\
\hline $\mathbf{4 . 2 6 6}$ & 2.944 \\
\hline
\end{tabular}

Note that the flow rate is changing according to the change of concentration due to the change in the hydrodynamic fluid pressure, but since the membrane dimensions and material are identical in every test the proportional constant governing the relation between the flow rate and the concentration is the membrane permeability. The data in table (1) is represented graphically in figure (18) using logarithmic scale for concentration.

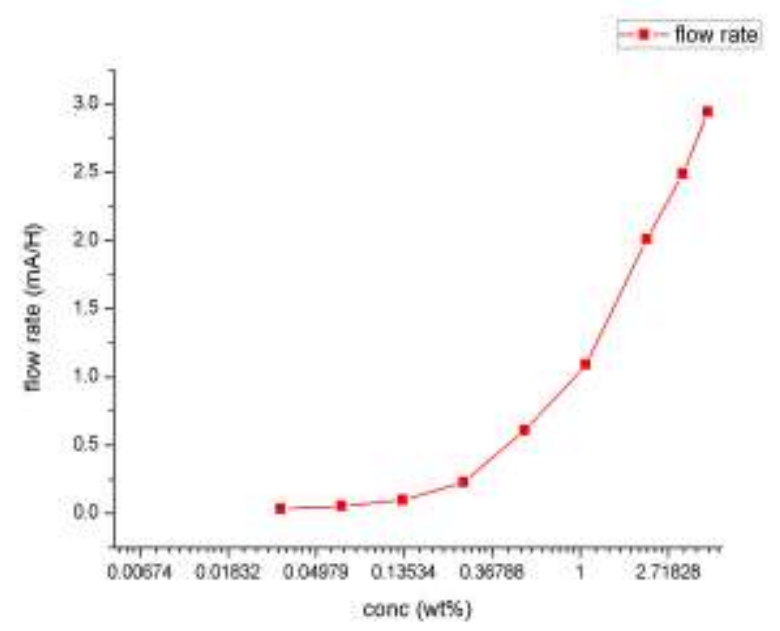

Figure (18): Flow rate as function of concentration.

\section{Empirical System Equation:}

The parameters involved in the permeability process can be divided into three main groups:

- Parameters related to the nature of the measurement technique which are the viscosity and concentration of the electrolyte.

- Parameters related to the permeability process itself which is the flow rate through the membrane.

- Parameters related to the sample under investigation which are the sample thickness and area.

\section{Viscosity:}

The viscosity of the solution must be involved in our equation as many other permeability equations, however most equations and according to their measurement mechanism use the dynamic viscosity $(\mu)$ rather than the kinematic viscosity $(v)$. The reason of this is based on the fact that, these systems measure the permeability by the aid of fluid flow through the sample under investigation. Unlike these systems our measurement technique uses the hydrodynamic fluid pressure, for this reason we going to use the kinematic viscosity instead of the dynamic viscosity.

The value $1.0244 \times 10^{-6} \mathrm{~m}^{2} / \mathrm{s}$ is taken as the average value for this parameter in our equation for the calculation of permeability over the used range of concentrations.

\section{Flow rate:}

The flow rate in our system is calculated by $\mathrm{mA} / \mathrm{H}$ which is a non-standard unit. So we must convert our flow rate to another standard unit used for measuring the flow rate.

The relation between the system parameters can be expressed by the following three relations [10]:

$$
\begin{gathered}
1 C=6.24 \times 10^{18} e \\
1 \text { mole }=96,485.4 C \\
1 A=1 C / s
\end{gathered}
$$

(where $\mathrm{C}=$ Coulomb, $\mathrm{e}=$ electron charge, $\mathrm{A}=$ Ampere, $\mathrm{s}=$ second) 
This leads to the following relation:

$$
1 \mathrm{~mA} / \mathrm{H}=2.877 \times 10^{-12} \mathrm{~mol} / \mathrm{s}
$$

The previous figure (17) is redrawn after conversion to the IS units.

It's found that the best fit equation for the graph is :

$$
k=\frac{Q}{\left(1-e^{-v C d / A b)}\right)}
$$

Where,

$$
\begin{aligned}
& k \text { is the membrane permeability in } \mathrm{m}^{2} \\
& Q \text { is the flow rate in } \mathrm{mol} / \mathrm{sec}
\end{aligned}
$$

$v$ is the kinematic viscosity in $\mathrm{m}^{2} / \mathrm{s}$

$C$ is the concentration in $\mathrm{mol} / \mathrm{m}^{3}$

$d$ is the membrane thickness in $m$

$A$ is the membrane area in $m^{2}$

$b$ is a numerical constant of value $5.522372 \times 10^{-5}$

Now the modified pulsed technique for measuring permeability of thin polymer films is ready to be used for permeability measurements.

\section{Applications of the system:}

The control of the permeability of polymer films is important task in this work. A composite of PVA and nano copper (70 nm) was prepared as a film using the previously described casting method. The films are filled with the copper nano particles with 5, 10, and $20 \mathrm{wt} \%$ ratios. Figure (19) shows the obtained flow rate curves and table (3) represents the recorded permeability values for the three films using the system imperial equation.

Table (3): Permeability of copper filled PVA films

\begin{tabular}{|c|c|}
\hline $\begin{array}{c}\text { Filled copper ratio } \\
\text { (wt\%) }\end{array}$ & Permeability k $\left(\boldsymbol{m}^{\mathbf{2}}\right)$ \\
\hline $\mathbf{0}$ & $9.082 \times 10^{-12}$ \\
\hline $\mathbf{5}$ & $8.86 \times 10^{-12}$ \\
\hline $\mathbf{1 0}$ & $7.974 \times 10^{-12}$ \\
\hline $\mathbf{2 0}$ & $6.955 \times 10^{-12}$ \\
\hline
\end{tabular}

We can see that as the copper particles increase the permeability decrease compared to the pure PVA films (zero ratio in table 3). These results agree with the expected one where as the particles increase in the film more blocking in the flow direction occurs.

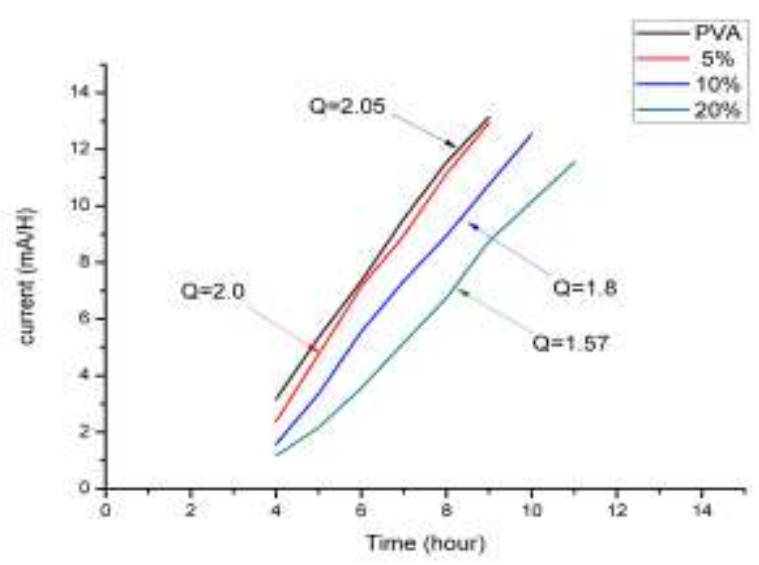

Figure (19): Flow rate for PVA membranes with different copper filling concentrations.

Another set of PVA films using the same preparation method but this time the films are filled with mico particles $(100-120 \mu \mathrm{m})$ of silicone oxide with 5,10 , and $20 \mathrm{wt} \%$ ratios.

The recorded permeability values for the three films using our system imperial equation are represented in table (4), while the flow rate curves are shown in figure (20).

Table (4): Permeability of silicon dioxide filled PVA films

\begin{tabular}{|c|c|}
\hline $\begin{array}{c}\text { Filled silicone oxide } \\
\text { ratio (wt \%) }\end{array}$ & Permeability k $\left(\boldsymbol{m}^{\mathbf{2}}\right)$ \\
\hline $\mathbf{0}$ & $9.082 \times 10^{-12}$ \\
\hline $\mathbf{5}$ & $1.2 \times 10^{-11}$ \\
\hline $\mathbf{1 0}$ & $9.259 \times 10^{-12}$ \\
\hline $\mathbf{2 0}$ & $7.265 \times 10^{-12}$ \\
\hline
\end{tabular}

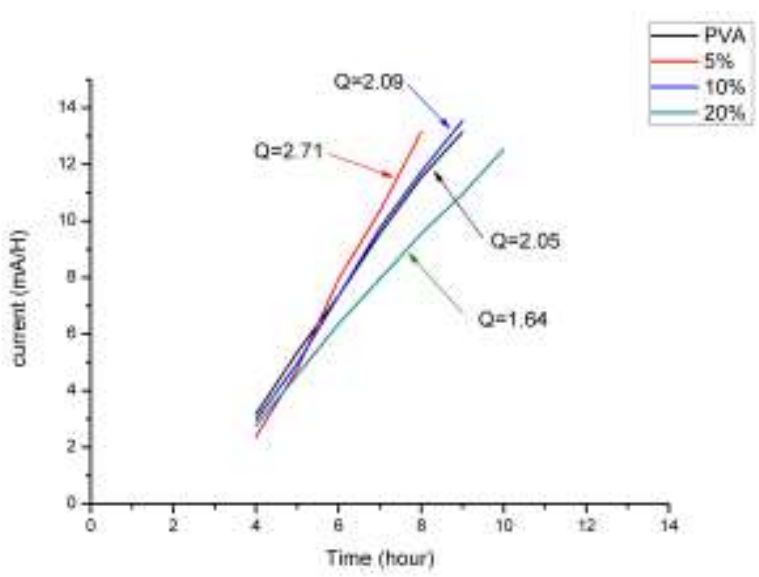

Figure (20): Flow rate for PVA membranes with different silicon dioxide filling concentrations. 
From figure 19 it is clear that the flow rate for the $5 \mathrm{wt} \%$ filled films is greater than the pure PVA one, which is on the contrary of the expected result.

A theoretical explanation of what can cause this unexpected result assume that the filled particles in the micro scale added new voids between the polymer and the particle surface allowing the flow to be easier through this voids. However another factor is causing the flow rate to be decreased which is the blocking effect of the particle itself.

Thus the permeability depends on two factors work in opposite ways. The first factor, which is the voids allowing more flow rate and hence increased permeability. The second factor which is the blocking effect due to the particle volume leading to reduced flow rate and hence decreased permeability. The obtained flow rate is the net of the above two factors.

Scanning Electron Microscope image supports the discussed theoretical principle above is shown in figure (21).

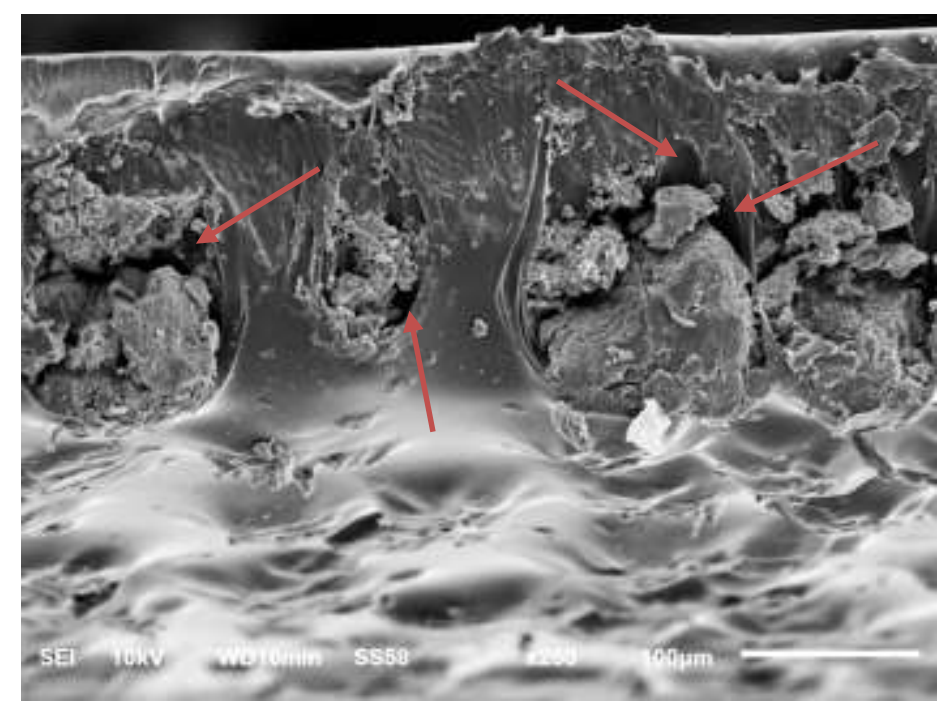

Figure (21): Red arrows aim to the voids around the silicone oxide micro particles

\section{CONCLUSION}

Permeability of membranes has great importance where membranes are of wide industrial and medical applications. Several techniques are designed to measure the permeability of membranes. Through these methods, fluids are forced to flow across the membrane by applying high pressure. By measuring the pressure difference, membrane surface area, thickness and flow rate of fluid the permeability is calculated.

Recently polymer membranes became one of the basic members of preamble materials. The main disadvantage that restricts the applicability of polymer membranes is there weak mechanical resistance against high pressures. The current work represented great modifications for the permeability measuring techniques. This modification is concluded in addition of ionic salt to water and evaluating the flow rate through the polymer membrane by direct measurement of electrolysis current behind the membrane. Several problems are raised during the establishment of this idea, technical solutions were applied. Finally, applying pulsed potential difference on the used graphite electrodes was an ideal answer for many side problems. By this answer it was possible to write a form for imperial equation correlates the flow rate across membrane, and hence the permeability, with the electrolyte current. This qualified measuring technique could be used to measure permeability of PVA membranes. It could, with aid of the modified technique, to change the permeability of PVA membranes by adding copper nano particles for decreasing permeability and adding silicone Dioxide micro particles to the PVA for increasing permeability of the membranes. Images by SEM supported the mechanism of controlling permeability that assumed by the authors.

\section{REFERENCES:}

[1] - Angelo Basile, Suzana Pereira Nunes, "Advanced membrane science and technology for sustainable energy and environmental applications", [ Woodhead Publishing] , pp:3-4 (2011)

[2] - Richard W. Baker, "Membrane Technology and applications" $2^{\text {nd }}$. Ed. , Membrane Technology and Research Inc,[John Wiley\& Sons Ltd, New York] pp. 15-16 (2000) 
[3] - Ali R. Zand, Yuri Sikorski, Matthew S. Sanders,

Homayun K. Navaz, "A Simple Laboratory Experiment for the Measurement of Single Phase Permeability", Journal of

Physics and Natural Science, vol. 1, Issue 2,(2007).

[4]- Thomas W. Engler, "Fluid Flow in Porous Media",

[Petroleum Engineering 524, New Mexico Tech.], pp: 3-12,

(2010)

[5] - Reza Masoodi , Krishna M. Pillai , "Wicking in Porous Materials - Traditional and Modern Modeling Approaches" , CRC Press - Taylor \& Francis Group , pp:13, (2013)

[6] - ENTEGRIS , INC. , "Properties and Characteristics of Graphite for Semiconductor Industry", pp: 19, (2013)

[7] - Measurement Computing Corporation , " Data Acquisition Handbook " , www.Mccdaq.com , pp: 31 , (2012)

[8] - John O’M. Bockris, Amulya K. N. Reddy, "Modern Electrochemistry", Second Edition, KLUWER ACADEMIC PUBLISHERS ,pp:429, (2002)

[9] - Bagotsky V. S. , "Fundamentals of Electrochemistry", Second Edition, [John Wiley \& Sons, New York - London] pp: 79 , (2006)

[10] - Allen J. Bard , Larry R. Faulkner , "Electrochemical Methods Fundamentals and applications" , [John Wiley \& Sons, Inc., New York - London] ,pp:4 , (2001) 